\title{
Phylogenetic relationship of equine Actinobacillus species and distribution of RTX toxin genes among clusters
}

\author{
Peter KUHNERT $^{\mathrm{a} *}$, Hélène BERTHOUD ${ }^{\mathrm{a}}$, Henrik CHRISTENSEN ${ }^{\mathrm{b}}$, \\ Magne BISGAARD ${ }^{\mathrm{b}}$, Joachim FREY ${ }^{\mathrm{a}}$ \\ ${ }^{a}$ Institute of Veterinary Bacteriology, Laenggass-Str. 122, University of Bern, \\ 3012 Bern, Switzerland \\ ${ }^{\mathrm{b}}$ Department of Veterinary Microbiology, The Royal Veterinary and Agricultural University, \\ 1870 Frederiksberg C, Denmark
}

(Received 16 August 2002; accepted 2 December 2002)

\begin{abstract}
Equine Actinobacillus species were analysed phylogenetically by 16S rRNA gene (rrs) sequencing focusing on the species Actinobacillus equuli, which has recently been subdivided into the non-haemolytic A. equuli subsp. equuli and the haemolytic A. equuli subsp. haemolyticus. In parallel we determined the profile for RTX toxin genes of the sample of strains by PCR testing for the presence of the A. equuli haemolysin gene aqx, and the toxin genes apxI, apxII, apxIII and apxIV, which are known in porcine pathogens such as Actinobacillus pleuropneumoniae and Actinobacillus suis. The rrs-based phylogenetic analysis revealed two distinct subclusters containing both A. equuli subsp. equuli and A. equuli subsp. haemolyticus distributed through both subclusters with no correlation to taxonomic classification. Within one of the rrs-based subclusters containing the A. equuli subsp. equuli type strain, clustered as well the porcine Actinobacillus suis strains. This latter is known to be also phenotypically closely related to A. equuli. The toxin gene analysis revealed that all A. equuli subsp. haemolyticus strains from both $r r s$ subclusters specifically contained the aqx gene while the A. suis strains harboured the genes apxI and apxII. The aqx gene was found to be specific for A. equuli subsp. haemolyticus, since A. equuli subsp. equuli contained no $a q x$ nor any of the other RTX genes tested. The specificity of $a q x$ for the haemolytic equine A. equuli and ApxI and ApxII for the porcine A. suis indicates a role of these RTX toxins in host species predilection of the two closely related species of bacterial pathogens and allows PCR based diagnostic differentiation of the two.
\end{abstract}

Actinobacillus / horse / RTX toxin / phylogeny / diagnostic

\section{INTRODUCTION}

Equine bacteria of the family of Pasteurellaceae are of clinical importance, since they may cause disease in horses and are found in infected wounds of humans bitten by horses $[4,5]$. Most of these bacteria fit into the genus Actinobacillus, and

\footnotetext{
* Correspondence and reprints

Tel.: (41) 31 6312485; fax: (41) 31 6312634; e-mail: peter.kuhnert@vbi.unibe.ch
} 
the majority of them belongs to the species Actinobacillus equuli. However, phenotypically this species shows a high variability including haemolytic activity. Haemolytic strains of $A$. equuli seem to be part of the normal flora of the equine oral cavity, but are also isolated from tracheal washes, indicating a preference for the respiratory tract [9]. These organisms also seem to represent opportunistic pathogens since they have been found associated with various clinical pictures in horses including respiratory infections, septicaemia, metritis, mastitis, arthritis, endocarditis, meningitis or stillbirth [3, 18]. Nonhaemolytic strains of $A$. equuli also seem to belong to the normal flora of the oral cavity and alimentary tract of horses. In addition they are frequently reported as an opportunistic pathogen, mainly in young animals. The most prominent clinical condition associated with these strains is "sleepy foal disease", an acute septicaemia of foals with purulent nephritis which eventually becomes chronic in the form of "joint ill", a polyarticular arthritis. The haemolytic strains of $A$. equuli, previously known as Bisgaard Taxon 11, have only been reported from horses but not specifically associated with "sleepy foal disease". The haemolytic strains of A. equuli led to the proposal of two subspecies: A. equuli subsp. haemolyticus and A. equuli subsp. equuli [5].

The factor responsible for the haemolytic phenotype of $A$. equuli strains has recently been described to be the Aqx protein, a member of the RTX toxin family [2]. Many representatives of RTX toxins are found in Pasteurellaceae [7]. The aims of the present investigation were to examine the phylogenetic relationship of a collection of strains of $A$. equuli and assess the distribution of the RTX toxin genes $a q x$ and apxIA, apxIIA, apxIIIA and apxIV in phylogenetic clusters established.

In order to get a broader view on the distribution of $a q x$ as well as to consider the recent classification of $A$. equuli into two subspecies we also carried out a thorough study on a variety of equine isolates. These included Pasteurella caballi, equine isolates of Actinobacillus lignieresii (recently classified as Actinobacillus genomospecies 1 [4]) as well as representatives of Bisgaard taxon 9 (recently classified as Actinobacillus arthritidis and Actinobacillus genomospecies 2 [4]) and Bisgaard taxon 10. In order to compare these findings with the phenotypically and phylogenetically closely related A. suis [1, 6], several porcine isolates of this species were included in addition to type strains of taxa investigated.

\section{MATERIALS AND METHODS}

\subsection{Bacterial strains and growth}

Type and reference strains of the different species were received from the American Type Culture Collection (ATCC), and the Culture Collection at the University of Göteborg (CCUG) and were identified accordingly in Figure 1. Field strains were isolated from equidae and pigs by the diagnostic units of the Institutes of Veterinary Bacteriology of the Universities Bern and Zurich (Switzerland), the Department of Disease Control and Biosecurity, National Veterinary Institute (Sweden), and the Department of Veterinary Microbiology at the Royal Veterinary and Agricultural University (Denmark) (Fig. 1). They were characterized and identified phenotypically by conventional methods in the laboratory where they had been kept. Strains

Figure 1. Phylogeny based on $16 \mathrm{~S}$ rRNA gene sequence, haemolytic phenotype and RTX toxin gene distribution of equine and porcine isolates of $A$. equuli and related species. Bootstrap values of 500 repeated experiments are indicated as percent at the major branches. The bar represents $2 \%$ sequence divergence.

* Strain negative by PCR, but positive by Southern blot. 


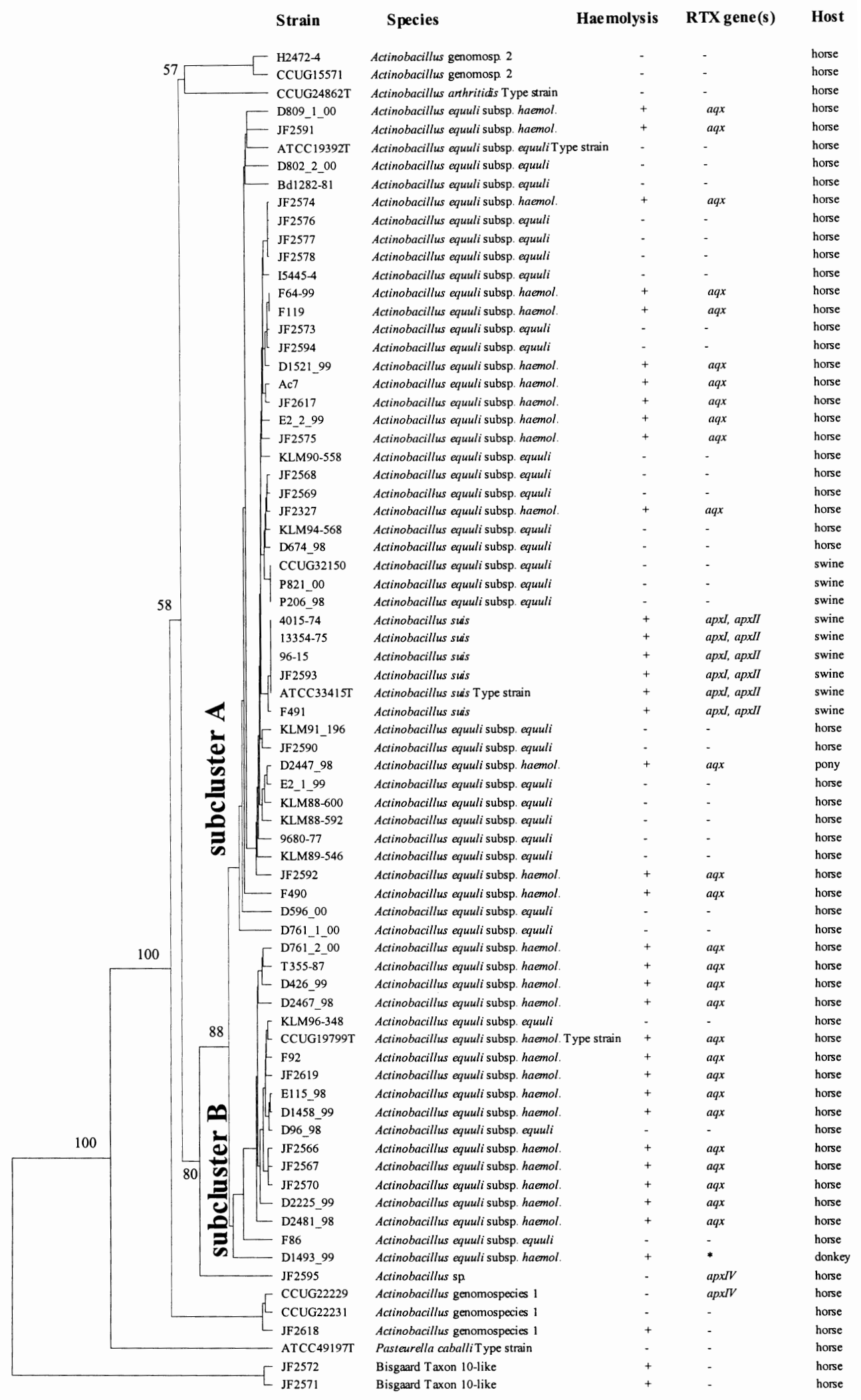


were grown on 5\% sheep blood agar plates at $37{ }^{\circ} \mathrm{C}$ overnight. Agar plates were prepared by overlaying Blood-AgarBase (Oxoid, Hampshire, UK) plates with Trypticase-Soy-Agar (BBL, Beckton Dickinson, Cockeyesville, MD, USA) supplemented with $0.1 \% \mathrm{CaCl}_{2}$ and $5 \%$ sheep blood.

\subsection{DNA isolation and PCR analysis}

Genomic DNA was extracted using either the QIAamp Tissue kit (Qiagen, Basel, Switzerland) or the Genomic DNA isolation kit PUREGENE ${ }^{\circledR}$ (Gentra Systems, Minneapolis, USA). Specific Aqx $\mathrm{PCR}$, based on the aqxA gene, was done as described by Berthoud et al. [2], while specific ApxI, ApxII and ApxIII PCR, amplifying apxIA, apxIIA, apxIIIA plus a small part of the corresponding $\mathrm{C}$ genes, was carried out as described by Frey et al. [8]. Specific ApxIV PCR was finally done as reported by Schaller et al. [17].

\subsection{S rRNA gene sequencing and phylogenetic analysis}

Determination of the 16S rRNA gene sequences in both directions was done as described previously [12, 14]. Sequence alignment and editing were done with the software Sequencher ${ }^{\mathrm{TM}}$ (Gene Codes Corporation, Ann Arbor, MI, USA). Sequence comparisons, cluster analysis and tree construction using UPGMA were done in BioNumerics Ver. 3.00 (Applied Maths, Kortrijk, Belgium).

\subsection{Southern-blot analysis}

Genomic DNA of bacterial strains was digested by EcoRI and size separated on a $0.7 \%$ agarose gel. Southern-blotting was done by alkaline transfer of DNA onto positively charged nylon membranes using an LCB 2016 Vacu Gene vacuum blotting pump (Pharmacia LKB biotechnology AB, Bromma, Sweden). After hybridization with $a q x$-specific probes [2], blots were washed at room-temperature two times, 5 min each, with $2 \times$ SSC- $0.1 \%$ SDS (1× $\mathrm{SSC}$ is $0.15 \mathrm{M} \mathrm{NaCl}$ plus $15 \mathrm{mM}$ sodium citrate), followed by two times washes at room-temperature, 15 min each, with $0.2 \times$ SSC-0.1\% SDS. Signals were detected by chemiluminescence using the CDPStar substrate (Roche Applied Science, Rotkreuz, Switzerland) followed by exposition to X-ray films.

\section{RESULTS}

Figure 1 summarizes the results of our analyses. Strains of the species $A$. equuli and $A$. suis formed a mono-phyletic group. This cluster can be divided into two subclusters matching the rrs sequences of the type strains of $A$. equuli subsp. equuli (ATCC19392) and A. equuli subsp. haemolyticus (CCUG19799), respectively. The $a q x$ gene was found in both subclusters $\mathrm{A}$ and $\mathrm{B}$ and specifies the haemolytic strains. No correlation between haemolysis and phylogenetic grouping was observed, even though within subcluster A out of 37 equine isolates 14 were haemolytic (38\%) whereas in subcluster B, 15 of 18 equine strains were haemolytic $(83 \%)$. The strain D1493_99 isolated from a donkey was negative for $a q x$ by PCR, but showed a strong hybridisation signal at high stringency in a Southern-hybridisation with an aqxCA specific probe. The EcoRI DNA fragment which reacted with this probe was larger than the EcoRI fragment reacting with the same probe in equine strains of A. equuli subsp. haemolyticus, indicating the presence of a variant of the aqx operon (data not shown). Within subcluster A clustered also 3 non-haemolytic porcine A.equuli as well as the haemolytic $A$. suis strains. The equine isolates A. equuli subsp. haemolyticus always contained the aqx gene and neither apxI nor apxII genes, while haemolytic swine isolates contained always apxI and apxII but never $a q x$. No $a q x$ genes could be detected in species other than A. equuli. 
Isolate JF2618 clustered together with the strains CCUG22231 and CCUG22229, belonging to Actinobacillus genomospecies 1. Strain CCUG22229 as well as JF2595 showed a positive PCR reaction for the apxIV. Strain JF2595 forms a branch of its own, most closely related to the $A$. equuli cluster showing in absolute numbers 98\% similarity of its rrs sequence to its nearest neighbour. It shares still $97 \%$ rrs sequence similarity with the other apxIV containing strain CCUG22229.

Actinobacillus arthritidis and genomospecies 2 as well as Pasteurella caballi clustered separately but showed neither haemolysis nor the presence of any of the investigated RTX toxin genes. Similar observations were made for the haemolytic Bisgaard taxon 10-like isolates which form an outgroup in the tree.

None of the equine nor porcine strains analysed showed up positive for the apxIII genes.

\section{DISCUSSION}

To investigate the relation between phylogeny and haemolytic phenotype we analysed the 16S rRNA gene sequences ( $r r s$ ) of field and reference strains of $A$. equuli and related species isolated mainly from horses and assessed haemolysis on sheep blood agar. In parallel, we defined the RTX toxin gene profile of these strains by PCR analysis. We limited our investigation to the aqx gene described for A. equuli subsp. haemolyticus, and apxI, apxII, apxIII and apxIV which are present in A. pleuropneumoniae and other members of the Pasteurellaceae family, including A. suis [16].

Besides the main cluster formed by the A. equuli isolates other phylogenetic groups can be discerned. Strains of Actinobacillus genomospecies 1 [4] clustering together have formerly been described as equine Actinobacillus lignieresii, a species which is phenotypically closely related to
A. pleuropneumoniae. This close phenotypic relationship is supported by the earlier description that strain CCUG22229 was typed as A. pleuropneumoniae serotype 3 [4]. Surprisingly this strain as well as JF2595 showed a positive PCR reaction for the apxIV gene which is considered as specific marker for A. pleuropneumoniae [17], indicating a potential horizontal gene transfer of apxIV or a similar gene among certain rare species of Pasteurellaceae. The haemolytic determinant of JF2618 however, could not be attributed to any of the investigated RTX genes. The same holds true for the Bisgaard Taxon 10-like strains. They both showed haemolysis on sheep blood agar plates but none of the 5 RTX toxin genes we looked for was detected in these strains. Therefore, the haemolytic determinant of these strains remains unknown.

In the phylogenetic group containing A. arthritidis and Actinobacillus genomospecies 2 the absence of the analysed RTX genes corresponds with the non-haemolytic phenotype of the strains.

No apxIIIA genes were found in the strains analysed. The ApxIII determinant as well as the very similar Pax RTX toxin have so far only been found associated with porcine isolates like Actinobacillus pleuropneumoniae, Actinobacillus rossii or Pasteurella aerogenes [13, 16].

The main focus of our study was on the A. equuli isolates which formed two phylogenetic clusters, one of them including also the A. suis isolates. These two subclusters, characterized by a major branching point with a boots-trap value of $88 \%$ (Fig. 1), correspond to previously reported phylogenetic subcluster around the A. equuli type strain ATCC19392 and the subcluster characterized by strain CCUG19799 tentatively named $A$. equuli-like [2]. Three nonhaemolytic porcine isolates clustered within subcluster A and probably represent A. equuli subsp. equuli previously reported from pigs [3]. Genuine porcine isolates of A. suis also clustered within subcluster 
A confirming previous observations [6], but formed a small group of their own within the tree, phenotypically corresponding to haemolysis, unique negative reactions in $\mathrm{D}(-)$ mannitol and formation of a yellowish pigment on blood agar. No correlation could be found between the two clusters and the recent classification of A. equuli into the two subspecies $A$. equuli subsp. equuli and $A$. equuli subsp. haemolyticus. However, all A. equuli subsp. haemolyticus contained the recently described haemolysin gene aqx, which confers the haemolytic phenotype of this subspecies [2]. No apx genes were detected in any of the A. equuli isolates. On the other side the haemolytic species $A$. suis always contained the apxI and apxII genes but no $a q x$, which was only found in strains of equine origin. Therefore, the haemolytic phenotype common to A. suis and A. equuli subsp. haemolyticus is clearly mediated by different haemolysins, and toxin gene detection by PCR provides a good method to differentiate these phenotypically as well as phylogenetically so closely related species. Furthermore, the different toxin gene profiles of $A$. suis and A. equuli subsp. haemolyticus could be an indication that RTX toxins contribute to the host specificity of the two bacterial species $A$. suis and A. equuli subsp. haemolyticus containing the apx and the aqx genes, respectively. We could recently show, that the RTX toxins ApxI, ApxII and Aqx have host-cell specific cytotoxic activities [15]. In these experiments ApxI and ApxII showed a higher toxic activity towards swine lymphocytes compared to equine lymphocytes, and on the other side Aqx was more toxic for horse than for pig lymphocytes. Differences in the toxic activities are most probably due to specific binding of the toxin to receptors on the target cells and/or differences in the signaling cascade after toxin binding. This was shown for other RTX toxins like the leukotoxin Lkt of Mannheimia haemolytica which after binding to the LFA-1 receptor is toxic for bovine leuko- cytes but has no effect on porcine or equine lymphocytes [10, 11, 19].

Our investigations clearly show, that the haemolytic phenotype common to $A$. suis and A. equuli subsp. haemolyticus is due to different toxin genotypes which is conserved and associated with the host origin of the isolates. This fact indicates that previous reports on isolation of $A$. suis from horses represent misidentifications or at least misnaming, which can easily be avoided by specific PCR for toxin gene detection. This study therefore shows that RTX toxin analysis provides a good tool for identification of animal pathogens and the assessment of their virulence.

\section{ACKNOWLEDGEMENTS}

We are grateful to Susanna Sternberg and Max Wittenbrink for sending us strains. We thank Yvonne Schlatter for excellent technical help. This work was supported by grant no. 5002-57817 of the Priority Programme "Biotechnology" of the Swiss National Science Foundation and by the Research Fund of the Institute of Veterinary Bacteriology, Bern.

\section{REFERENCES}

[1] Bada R., Mittal K.R., Higgins R., Biochemical and antigenic relationships between porcine and equine isolates of Actinobacillus suis, Vet. Microbiol. 51 (1996) 393-396.

[2] Berthoud H., Frey J., Kuhnert P., Characterization of Aqx and its operon: the hemolytic RTX determinant of Actinobacillus equuli, Vet. Microbiol. 87 (2002) 159-174.

[3] Bisgaard M., Ecology and significance of Pasteurellaceae in animals, Zentralbl. Bakteriol. 279 (1993) 7-26.

[4] Christensen H., Bisgaard M., Angen O. Olsen J.E., Final classification of Bisgaard taxon 9 as Actinobacillus arthritidis sp. nov. and recognition of a novel genomospecies for equine strains of Actinobacillus lignieresii, Int. J. Syst. Evol. Microbiol. 52 (2002) 12391246.

[5] Christensen H., Bisgaard M., Olsen J.E., Reclassification of equine isolates previously reported as Actinobacillus equuli, variants of 
A. equuli, A. suis or taxon 11 of Bisgaard and proposal of two subspecies of A. equuli: $A$. equuli subsp. equuli and $A$. equuli subsp. haemolyticus, Int. J. Syst. Evol. Microbiol. 52 (2002) 1569-1576.

[6] Dewhirst F.E., Paster B.J., Olsen I., Fraser G.J., Phylogeny of 54 representative strains of species in the family Pasteurellaceae as determined by comparison of $16 \mathrm{~S}$ rRNA sequences, J. Bacteriol. 174 (1992) 20022013.

[7] Frey J., Kuhnert P., RTX toxins in Pasteurellaceae, Int. J. Med. Microbiol. 292 (2002) $149-158$

[8] Frey J., Beck M., Vandenbosch J.F., Segers R.P.A.M., Nicolet J., Development of an efficient PCR method for toxin typing of Actinobacillus pleuropneumoniae strains, Mol. Cell. Probes 9 (1995) 277-282.

[9] Jang S.S., Biberstein E.L., Actinobacillus suis-like organisms in horses, Am. J. Vet. Res. 48 (1987) 1036-1038.

[10] Jeyaseelan S., Hsuan S.L., Kannan M.S., Walcheck B., Wang J.F., Kehrli M.E., Lally E.T., Sieck G.C., Maheswaran S.K., Lymphocyte function-associated antigen 1 is a receptor for Pasteurella haemolytica leukotoxin in bovine leukocytes, Infect. Immun. 68 (2000) 72-79.

[11] Jeyaseelan S., Kannan M.S., Briggs R.E., Thumbikat P., Maheswaran S.K., Mannheimia haemolytica leukotoxin activates a nonreceptor tyrosine kinase signaling cascade in bovine leukocytes, which induces biological effects, Infect. Immun. 69 (2001) 6131-6139.

[12] Kuhnert P., Capaul S.E., Nicolet J., Frey J., Phylogenetic positions of Clostridium chauvoei and Clostridium septicum based on $16 \mathrm{~S}$
rRNA gene sequences, Int. J. Syst. Bacteriol. 46 (1996) 1174-1176.

[13] Kuhnert P., Heyberger-Meyer B., Nicolet J., Frey J., Characterization of PaxA and its operon: a cohemolytic RTX toxin determinant from pathogenic Pasteurella aerogenes, Infect. Immun. 68 (2000) 6-12.

[14] Kuhnert P., Frey J., Lang N.P., Mayfield L., A phylogenetic analysis of Prevotella nigrescens, Prevotella intermedia and Porphyromonas gingivalis field strains reveals a clear species clustering, Int. J. Syst. Evol. Microbiol. 52 (2002) 1391-1395.

[15] Kuhnert P., Berthoud H., Straub R., Frey J., Host specific activity of RTX toxins from haemolytic Actinobacillus equuli and Actinobacillus suis, Vet. Microbiol. 92 (2003) 161167.

[16] Schaller A., Kuhnert P., De La Puente V., Nicolet J., Frey J., Apx toxins in Pasteurellaceae species from animals, Vet. Microbiol. 74 (2000) 365-376.

[17] Schaller A., Djordjevic S.P., Eamens G.J., Forbes W.A., Kuhn R., Kuhnert P., Gottschalk M., Nicolet J., Frey J., Identification and detection of Actinobacillus pleuropneumoniae by PCR based on the gene apxIVA, Vet. Microbiol. 79 (2001) 47-62.

[18] Sternberg S., Brandstrom B., Biochemical fingerprinting and ribotyping of isolates of Actinobacillus equuli from healthy and diseased horses, Vet. Microbiol. 66 (1999) 53-65.

[19] Sun Y., Clinkenbeard K.D., Clarke C., Cudd L., Highlander S.K., Dabo S.M., Pasteurella haemolytica leukotoxin induced apoptosis of bovine lymphocytes involves DNA fragmentation, Vet. Microbiol. 65 (1999) 153-166. 